\title{
Three-dimensional photoacoustic imaging using fiber-based line detectors
}

\author{
Hubert Grün \\ Thomas Berer \\ Peter Burgholzer \\ RECENDT GmbH \\ Hafenstrasse 47-51 \\ Linz, Austria 4020
}

\author{
Robert Nuster \\ Günther Paltauf \\ Karl-Franzens-University \\ Department of Physics \\ Universitätsplatz 5 \\ Graz, Austria 8010
}

\begin{abstract}
For photoacoustic imaging, usually point-like detectors are used. As a special sensing technology for photoacoustic imaging, integrating detectors have been investigated that integrate the acoustic pressure over an area or line that is larger than the imaged object. Different kinds of optical fiber-based detectors are compared regarding their sensitivity and resolution in three-dimensional photoacoustic tomography. In the same type of interferometer, polymer optical fibers yielded much higher sensitivity than glass fibers. Fabry-Pérot glassfiber interferometers in turn gave higher sensitivity than MachZehnder-type interferometers. Regarding imaging resolution, the single-mode glass fiber showed the best performance. Last, threedimensional images of phantoms and insects using a glass-fiber-based Fabry-Pérot interferometer as integrating line detector are presented. () 2010 Society of Photo-Optical Instrumentation Engineers. [DOI: $10.1117 / 1.3381186]$
\end{abstract}

Keywords: photoacoustic imaging; integrating detectors; fiber-based detectors; three-dimensional imaging.

Paper 09354SSR received Aug. 19, 2009; revised manuscript received Feb. 10 2010; accepted for publication Feb. 10, 2010; published online Apr. 21, 2010.

\section{Introduction}

Photoacoustic imaging is an emerging technology in the field of biological and medical imaging. ${ }^{1}$ Early breast cancer detection is only one example for a promising medical application, as reported, e.g., by Manohar et al. ${ }^{2}$ A short pulse of electromagnetic radiation, e.g., a short laser pulse, illuminates a semitransparent sample. Depending on the local specific absorption rates (SARs) in the object, the electromagnetic energy is absorbed and causes thermoelastic expansion. This is the photoacoustic effect that was investigated by Alexander Graham Bell in $1880 .^{3}$ Thereby broadband ultrasonic wavesphotoacoustic signals - are launched and propagate through the sample. Due to the nonuniform distribution of absorbed energy in different areas of the object, these photoacoustic signals contain information about light absorption at ultrasonic resolution and hence combine the advantages of pure optical imaging (high contrast) and ultrasonic imaging (high spatial resolution).

Once the photoacoustic signals have propagated to the surface of the sample, they can be measured using, e.g., conventional point-like piezoelectric detectors or integrating detectors. Integrating detectors were introduced by Haltmeier et al. ${ }^{4}$ in 2004. Meanwhile, several types of integrating detectors have been realized, and measurement results were shown, e.g., by Burgholzer et al. ${ }^{5}$ using integrating area detectors and Paltauf et al. ${ }^{6}$ using a free-beam Mach-Zehnder interferometer as implementation of an integrating line detector. Another approach for integrating line detectors are fiber-based interferometers, which are described in detail in Sec. 2.

Address all correspondence to: Hubert Grün, RECENDT GmbH, Hafenstrasse 47-51, Linz, Austria 4020. Tel: 43-732-90155642; Fax: 43-732-90155618; E-mail: hubert.gruen@recendt.at
Measuring the temporal pressure variations at various positions outside the sample allows reconstruction of the initial pressure distribution. For point-like detectors, reconstruction methods are given, e.g., by $\mathrm{Xu}$ and Wang. ${ }^{7}$ Using integrating line detectors requires alternative reconstruction procedures. These algorithms mostly consist of two parts. In the first part, 2-D projection images of the initial pressure distribution are reconstructed. In the second part, the 3 -D pressure distribution is calculated by a subsequent inverse Radon transform. ${ }^{8}$ For the first part of photoacoustic image reconstruction, we use a time-reversal reconstruction method, ${ }^{8,9}$ which is known from other applications. ${ }^{10}$ The reconstruction based on time reversal is briefly described in Sec. 3 .

In the last section, we present the first 3-D photoacoustic images obtained with the combination of integrating fiberbased line detectors and a time-reversal reconstruction method. Compared to earlier work, where images obtained with a free-beam interferometer were presented (Holotta et al. $\left.{ }^{11}\right)$, the results shown in this paper are measured with fiberbased integrating line detectors. The fundamental idea of integrating line detectors for detecting photoacoustic signals is the same in both approaches, but the realization of the detector is quite different. The fiber-based approach is more sophisticated and allows better handling of the detector. Results of 3-D imaging of a bristle knot and an ant are given, and also the results concerning reproduction of shape and dimensions are discussed. Last, future improvements and developments to gain a higher sensitivity and allow faster imaging with fiberbased line detectors are discussed.

$1083-3668 / 2010 / 15(2) / 021306 / 8 / \$ 25.00$ (C) 2010 SPIE 


\section{Integrating Line Detectors}

Usually for photoacoustic imaging, point-like detectors for acquiring photoacoustic signals are used. These point-like detectors are typically realized by piezoelectric sensors, but also optical point detectors have been reported, e.g., by Zhang et al. ${ }^{12}$ In 2004, Haltmeier et al. ${ }^{4}$ introduced integrating detectors that integrate the pressure at least along one dimension. Possible detector geometries are planes, lines, or more complex geometries. ${ }^{13,14}$ For tomographic applications, integrating line detectors are more convenient than integrating plane detectors, as reported by Burgholzer et al. ${ }^{15}$ In practice, it is easier to build a setup with only one rotation axis. Therefore, it was proposed to fragment the planar detector into an array of line detectors. Signals received by such line detectors at time $t$ are integrals of pressure distribution over cylinders with radius $c \cdot t$, where $c$ is the speed of sound. Due to the integration of the pressure field along the line detector, the dimensionality of the 3 -D wave propagation is reduced to a 2-D problem, and the corresponding equation can be written as

$$
\left(\frac{\partial^{2}}{\partial t^{2}}-c^{2} \Delta_{x, y}\right) \bar{p}(x, y, t)=0
$$

where $\bar{p}$ is the pressure integrated in the $z$ direction along the line detector. This 2-D wave equation can be solved using the initial conditions

$$
\bar{p}(x, y, t=0)=\int p_{0}(x, y, z) \mathrm{d} z \text { and } \frac{\partial \bar{p}(x, y, t=0)}{\partial t}=0,
$$

with $p_{0}$ as initial pressure distribution.

Integrating line detectors can be made, for example, from stripes of a piezoelectric material (PVDF) or by use of optical detectors. A laser beam that is part of an interferometer has been utilized as an integrating line detector. ${ }^{6}$ The interferometer measures variations of the refractive index induced by the acoustic pressure (elasto-optic effect). Implementations of interferometric detectors as integrating line detectors can be based on either free or guided light beams. Our group has studied all these types of integrating line detectors and compared their sensitivity as reported by Nuster et al. ${ }^{16}$ The directivity of piezo line detectors was investigated and described by Paltauf et al. ${ }^{17}$ However, no tomography using piezo line detectors has been done so far. Free-beam Mach-Zehnder interferometers have been used for photoacoustic tomography, and results have been published by Paltauf et al. ${ }^{6,18}$ Fiberbased interferometers have been studied using either glass fibers ${ }^{19}$ or polymer fibers. ${ }^{13,20}$

A free laser beam as acoustic detector is completely transparent, both optically and acoustically. It can therefore disturb neither the incoming excitation light pulse nor the outgoing acoustic wave. ${ }^{21}$ A major drawback of free-beam detection is that the high resolution can be achieved only in the focal range of the laser beam. For photoacoustic microtomography ( $\mu$-PAT), a free-beam detector can be focused to permit high resolution. Paltauf et al. ${ }^{22}$ investigated the influence of the beam width and the varying beam diameter for focused line detectors. For small objects (in the range of mm up to a few $\mathrm{cm})$, the varying beam diameter does not have an influence on the resolution, as long as the object is located within the focal depth of the lens. For larger samples, fiber-based detectors are more appropriate. Due to the constant diameter of the fiber core along the whole fiber, the resolution does not depend on the position. Theoretically, the resolution is limited by the 9 - $\mu \mathrm{m}$ core diameter of single-mode fibers. For medical imaging, e.g., breast imaging, a constant high resolution along the whole detector is important. Thus, focused free-beam detectors are not the best choice in this application. Fiber-based detectors have another advantage: they can be moved around a sample quite easily, whereas moving free-beam detectors are more difficult to build because of the more complex optical setup. Therefore, fiber-based detectors are dedicated for medical imaging of bigger volumes with high resolution. Another important issue for medical applications is the laser safety: using a fiber-based detector, the laser is guided in the fiber, and therefore no laser-induced injury can happen. Up to now, the lower sensitivity of fiber-based detectors compared to free-beam detectors has been a severe disadvantage. ${ }^{16}$ Strategies for increasing the sensitivity of fiber-based detectors will be discussed later in this section. For medical application, both types of interferometric line detectors have considerable advantages compared to standard piezoelectrical detectors. Both detectors not only are insensitive against electrical interference but also show a better broadband frequency response compared to standard piezoelectrical detectors. Furthermore, optical line detectors have no electrical connection between sensor and measurement device-there is only an optical connection. Standards for medical devices require also an electrical insulation in the case of a technical failure to protect patients against electric shocks. Therefore, optical integrating line detectors are promising detectors for medical applications.

Fiber-based detectors were realized using two different types of optical fibers: with glass optical fibers (GOFs) and with polymer optical fibers (POFs). Both provide different advantages and disadvantages. One of the important parameters affecting the spatial resolution is the core diameter. The core diameter divided by the speed of sound of the core material limits the theoretically achievable temporal resolution. Therefore, in general, a smaller core diameter is better for achieving high spatial resolution. Single-mode glass fibers exhibit a $9-\mu \mathrm{m}$ core diameter, whereas conventionally available perfluorinated polymer fibers provide a core diameter of $50 \mu \mathrm{m}$ and more. Any interferometric acoustic detector requires some kind of active stabilization for maintaining the optimum operation point, even in the presence of ambient vibrations. In a conventional free-beam interferometer, the distance between the mirrors can be adjusted. For the fiberbased interferometers, where the mirrors are usually fiber Bragg gratings (FBGs), we need a wavelength-adjustable laser for this purpose. Such a tunable single-frequency fiber laser for interferometry was available only at a wavelength of $1550 \mathrm{~nm}$, at which conventional POF (e.g., PMMA fibers) with smaller diameters than $50 \mu \mathrm{m}$ exhibit strong damping. Therefore, we used perfluorinated POFs, which provide less damping at $1550 \mathrm{~nm}$. Another criterion is the sensitivity of the fibers. Due to the much better impedance matching of polymer fibers to the surrounding water, their sensitivity is higher than for glass fibers, where approximately $2 / 3$ of the incoming signal is reflected before reaching the core. Addi- 


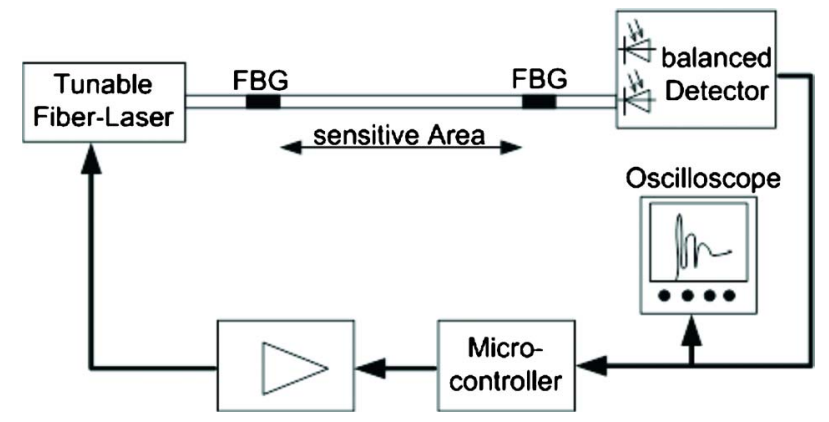

Fig. 1 A fiber-based Fabry-Pérot interferometer as integrating line detector for photoacoustic imaging. Two fiber Bragg gratings (FBGs) act as mirrors. The pressure waves are detected in the region between the fiber Bragg gratings.

tionally, the Young's modulus is much lower in POFs than in GOFs. Therefore, deformation of a POF is bigger than of a GOF applying the same pressure wave. As the strain optic coefficients are in the same order, this results in an enhanced change of refractive index and thus to higher signal amplitudes in the polymer fiber. ${ }^{23}$

Nuster et al. ${ }^{16}$ compared different types of integrating line detectors, concluding that the amplitude of a glass-fiber Mach-Zehnder interferometer is about 20 times smaller than the signal from the polymer-fiber Mach-Zehnder interferometer. The latter in turn has a slightly higher sensitivity than the used glass fiber-based Fabry-Pérot interferometer (FPI) with a finesse of approximately 18 . The sensitivity depends on the finesse-therefore, the glass-fiber FPI could be improved by another finesse. A Fabry-Pérot detector is sensitive only between the fiber Bragg gratings, which act as mirrors (see Fig. 1). Based on these findings, we decided to realize an integrating line detector with a fiber-based Fabry-Pérot interferometer with an FBG reflectivity of $81 \%$ and a distance between the FBGs of $11.5 \mathrm{~cm}$, resulting in a finesse of approximately 18 . A scheme of the setup can be seen in Fig. 1. Combining the advantages of the polymer fibers (higher sensitivity due to fewer losses) and the Fabry-Pérot interferometer (higher sensitivity due to the multibeam interferometer character) would improve the sensitivity tremendously. Future work will focus on realizing such a detector.

As described by Haltmeier et al. ${ }^{4}$ the length of the line detector (i.e., the distance between the FBGs) influences the dimensions of the objects that can be imaged. The length of the line detector has to be at least $\sqrt{8} \cdot D$, where $D$ is the diameter of a sphere that completely encloses the object and touches the detector. Using the described line detector, it is therefore possible to image objects that are smaller than $4 \mathrm{~cm}$ with this setup.

As mentioned earlier, the core diameter of the fiber $(9 \mu \mathrm{m}$ for single-mode glass fibers) is the theoretical resolution limit. Hence, the detection setup was designed for frequencies up to $240 \mathrm{MHz}$ to match this limit. This is only the theoretical estimation, disregarding factors like frequency-dependent attenuation or the resolution limit in dependence on the number of detectors and the increment between the detector positions. Up to now, no detailed studies about the frequency response of such glass-fiber-based Fabry-Pérot sensors as integrating line detectors have been carried out.

\section{Image Reconstruction}

The inverse photoacoustic problem aims to reconstruct the absorption density inside the sample from the acoustic pressure measured outside the illuminated sample. Using integrating line detectors, image reconstruction is a two-step process. Due to the integration of the pressure along the line detector, the imaging problem is reduced to a 2-D problem. The first step of reconstruction involves the calculation of a 2-D projection image, which could be done using different algorithms, e.g., back projection, ${ }^{24}$ Fourier transform, ${ }^{25}$ or timereversal reconstruction. ${ }^{8}$ After calculating a set of 2-D projection images from a range of directions, the inverse Radon transform is performed to reconstruct the initial 3-D pressure distribution. Kak and Slaney ${ }^{26}$ established an estimation stating that the number of projections should be roughly equal to the number of detector positions in each projection. Due to the long acquisition time for each projection image (approximately $20 \mathrm{~min}$ ), with our current setup only 25 projections (instead of 100) were measured for the images presented in this paper.

The results shown in this paper are calculated using the time-reversal reconstruction method, which is described in detail by Burgholzer et al. ${ }^{8}$ The idea of time reversal is to "rewind" the wave propagation. The pressure on the arbitrarily shaped detection surface is set to coincide with the measured data in temporal order. Time-reversal reconstruction is based on the invariance of the homogeneous wave equation under the transformation $t \rightarrow-t$, which is known from many other practical applications like material analysis, hydrodynamics, undersea communications, and medicine. ${ }^{27}$ Using this reconstruction method, the time-reversed field is directly calculated with a second-order embedded boundary method by retransmitting the measured pressure on the detector positions in reversed temporal order. This second-order embedded boundary method is described in detail also in Ref. 8.

For our 2-D imaging problem, this results in a simple reconstruction algorithm. A 2-D matrix is defined, and for each detector position around the sample, an element in this matrix is assigned. For every time step, the detected pressure is fed back in a time-reversed order at each detector position. The wave propagation is calculated, and after a certain time $T$, the initial pressure distribution is achieved. One advantage of this method is the possibility to compensate spatially varying speed of sound, e.g., bones embedded in tissue. A preliminary approach, first simulations, and a simple experiment were reported by Grün et al. ${ }^{28}$ for heterogeneous media. The speed of sound was assumed to be constant for the reconstruction of the presented results in this paper.

\section{Two-Dimensional and Three-Dimensional Imaging Results}

Since the first fiber-based setup was described, ${ }^{9}$ a semiautomatic setup has been developed. First, 2-D projection images were obtained using a bristle knot as simple phantom. Twodimensional projection images using the glass-fiber FabryPérot detector ${ }^{19}$ and the polymer-fiber Mach-Zehnder interferometer ${ }^{20}$ were presented. In Sec. 4.1, the setup, illumination conditions, and detector positions are described in detail. For these 2-D images of the bristle knot, only one projection at an angle of 0 deg was acquired. In Fig. 2, the 


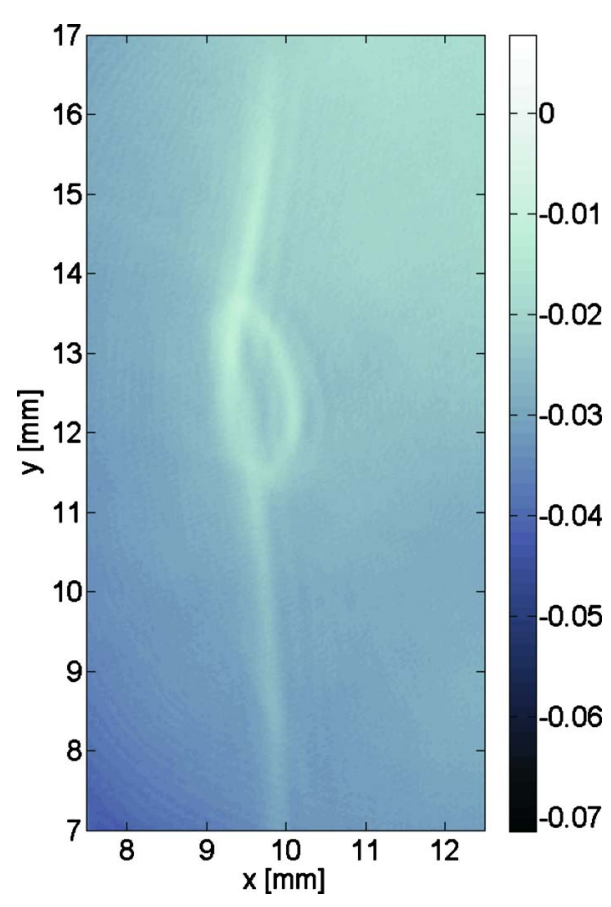

Fig. 2 Two-dimensional projection image of a bristle knot measured with a glass-fiber Fabry-Pérot interferometer. Some artifacts with the same shape of the object arise around the object. These artifacts are attributed to reflections in the glass fiber, as described in the text.

reconstructed 2-D projection image measured with the glassfiber Fabry-Pérot detector can be seen. For comparison, the same object was measured with a polymer-fiber MachZehnder interferometer (Fig. 3). One can recognize that the image taken with the glass fiber is slightly sharper than the image acquired with the polymer fiber. This may be due to the much bigger core diameter of the polymer fiber-as described in Sec. 2, a bigger core diameter influences the spatial resolution negatively. Looking closer at Fig. 2, some artifacts can be identified. These artifacts have the same shape as the imaged object and might be caused by reflections inside the glass fiber. These reflections arise from the acoustic impedance mismatch of the fiber core and cladding made of glass and the surrounding coating made of polymer. Reflections could be minimized by the use of matching layers between glass and the polymer coating. Another difference between both images is the contrast. Using the polymer fiber-based integrating detector, the contrast of the reconstructed image is higher than using the glass fiber-based detector. This might be due to the slightly better sensitivity of the polymer MachZehnder line detector.

Both fiber-based detector types are well suited as integrating line detectors for photoacoustic imaging. Due to its easier handling and higher stability, we chose the glass-fiber FabryPérot detector for the first 3-D photoacoustic imaging experiments.

\subsection{Three-Dimensional Imaging of a Bristle Knot}

First, 3-D measurements on a bristle knot (similar to Refs. 19 and 20) were made. The diameter of such a bristle varies between $150 \mu \mathrm{m}$ and $250 \mu \mathrm{m}$ due to its slightly conical shape. Because of the almost black color, the bristle is a good

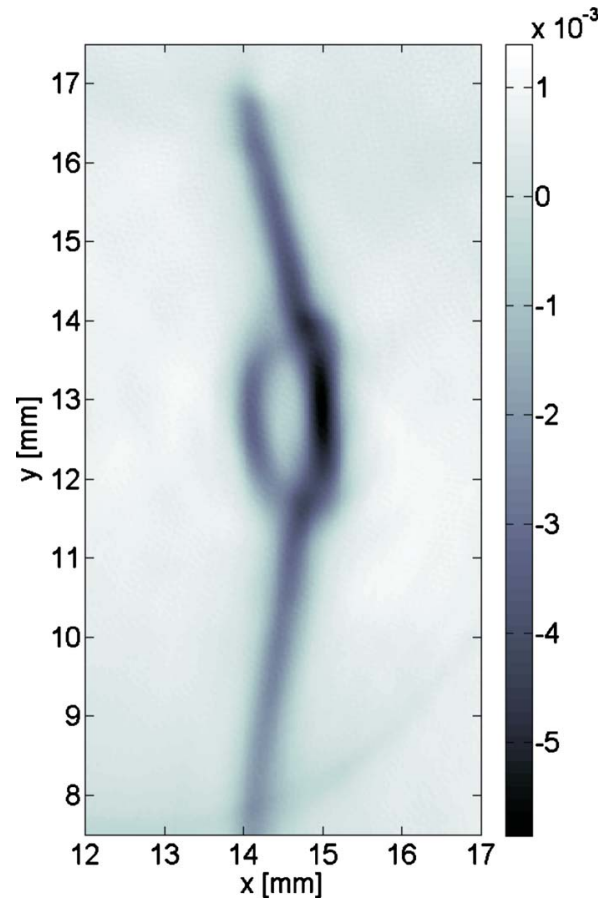

Fig. 3 A 2-D projection image acquired with the polymer-fiber MachZehnder detector. Compared to Fig. 2 this image appears more blurred and the structures are broadened, but the contrast is higher than in Fig. 2.

absorber at the used excitation wavelength $(532 \mathrm{~nm})$. Before mounting on the sample holder, the bristle was formed into a knot (or loop) with a diameter of approximately $800 \mu \mathrm{m}$, as can be seen later in Fig. 6 on the left side. The bare bristle knot was fixed in a clamp on the sample holder with no additional immobilization and immersed in a tank filled with water without any scattering medium.

For excitation, a frequency-doubled Nd:YAG $(532 \mathrm{~nm})$ pulse laser (pulse duration: $6 \mathrm{~ns}$, repetition rate: $20 \mathrm{~Hz}$ ) was used. The laser beam was split into two paths to excite the sample from two sides at an angle of about $80 \mathrm{deg}$, as depicted in Fig. 4. After splitting, the two beams were expanded to a diameter of approximately $20 \mathrm{~mm}$ to excite the whole sample at once. The pulse energy was adjusted to keep the radiant exposure below the allowed maximum value for biological tissue $\left(20 \mathrm{~mJ} / \mathrm{cm}^{2}\right.$ for using $\left.532 \mathrm{~nm}\right)$ to show the applicability for medical purposes.

A total of 262 detector positions arranged in a circle around the object (see Fig. 5) were used for collecting photoacoustic signals for calculating the 2-D projection. The diameter of the detection curve was $20 \mathrm{~mm}$. Thus, the distance between the object and the detector was in the range of 3 to $8 \mathrm{~mm}$, depending on the relative detector position. According to Kak and Slaney, ${ }^{26}$ the estimated spatial resolution in the 2-D projection-for this amount of detector positions and this radius of the detection curve-is approximately $110 \mu \mathrm{m}$, which is sufficient for the bristle knot. This showed that not all 262 detector positions are necessary, and therefore we reduced the amount of detector positions to 200 for imaging the ant. For a 2-D projection image, the fiber-based line detector was moved around the object. For calculating the 3-D 


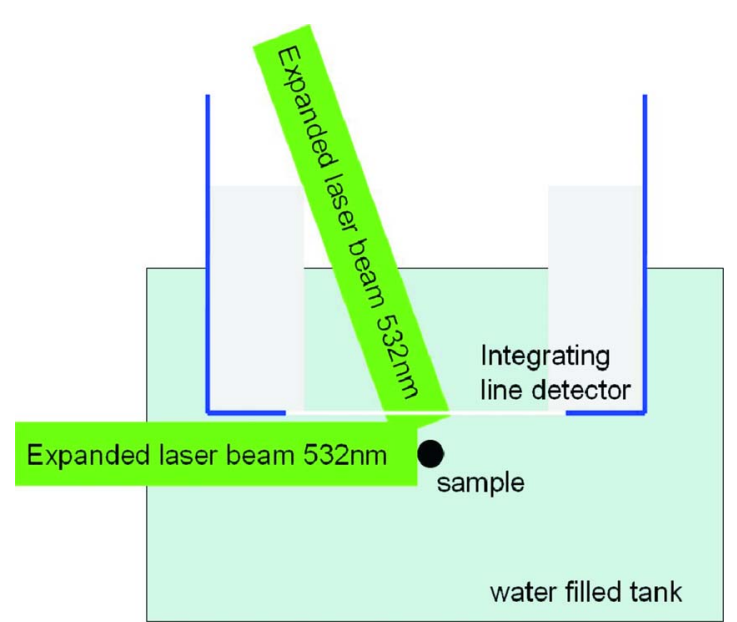

Fig. 4 Schematic of the sample illumination (top view). Illumination is done from two sides to achieve a homogeneous excitation. By using a (transparent) fiber-based line detector, it is possible to illuminate the sample through the detector.

image, 25 such projections with an angle increment of $7.2 \mathrm{deg}$ were scanned. As mentioned in Sec. 3, these are much fewer projections than necessary-but due to the long acquisition time per projection, this compromise had to be made. For acquiring 2-D projections at different angles, the object was moved around the rotation axis, as depicted in Fig. 5. Due to the low sensitivity as described earlier, we averaged the signals 64 times at each detector position, hence the averaged acquisition time per projection angle was about $20 \mathrm{~min}$, including the time where the line detector moves from one position to the next.

As mentioned in Sec. 2, Haltmeier et al. ${ }^{4}$ described a geometrical criterion requiring that the minimal length of the integrating line detector has to be $\sqrt{8} \cdot D$, where $D$ is the diameter of a sphere that completely encloses the object and touches the detector. The fiber-based line detector used had a sensitive length of $11.5 \mathrm{~cm}$. Therefore, objects with a size up to $4 \mathrm{~cm}$ could be imaged with this sensor. This is also the

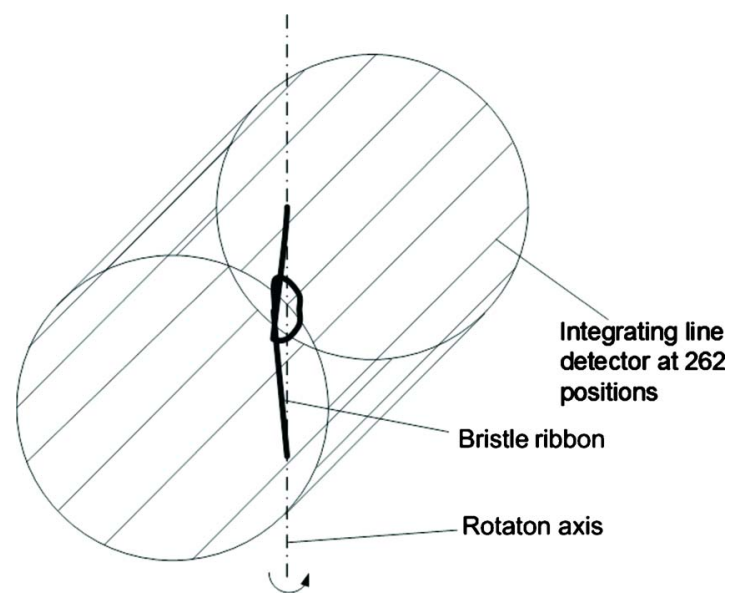

Fig. 5 Several detector positions arranged in a circle around the object for acquiring data for one projection image. 262 detector positions were used for imaging the bristle knot.
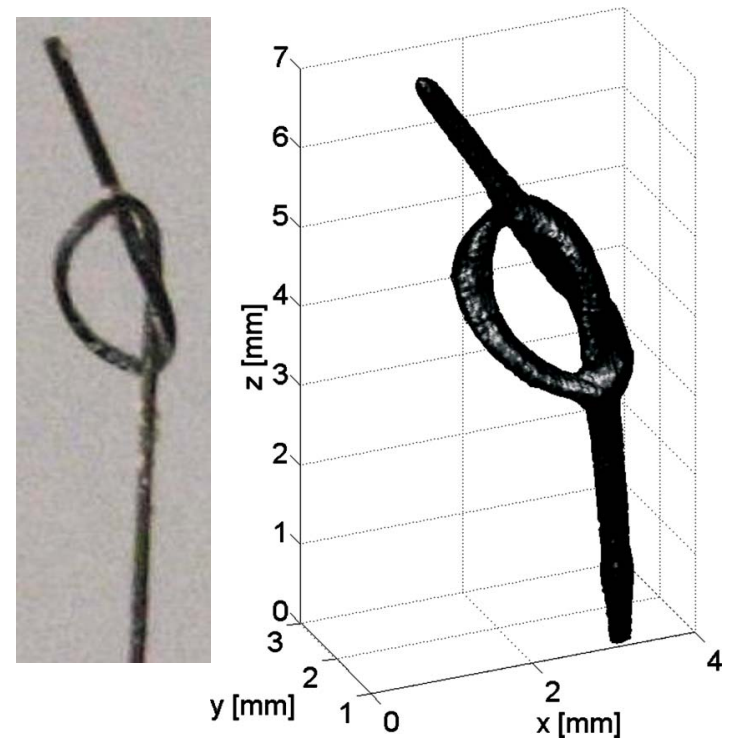

Fig. 6 A photograph of the bristle knot can be seen on the left. The diameter of the slightly conical bristle varies between 150 and $250 \mu \mathrm{m}$. The diameter of the loop is approximately $800 \mu \mathrm{m}$. Right: The 3-D reconstruction of the bristle knot from 25 projection images, each reconstructed from signals at 262 detector positions. The shape and the dimensions are reproduced well. Few artifacts occur because of the low number of projections used.

maximum object size in the current lab scanner. But the glassfiber-based line detectors can be easily scaled up for imaging bigger volumes, e.g., breast imaging, where a detector with a length of approximately $50 \mathrm{~cm}$ will be satisfactory.

The reconstructed volume of the bristle knot is depicted in Fig. 6. Although only 25 projections were used for the reconstruction of the volume, the shape and dimensions are reproduced well. Some deformations arise in the reconstructed volume-at the loop, a dent, and at the bootleg, a knob can be seen. These artifacts should vanish if more than 25 projections are used for reconstruction. Also, readjusting the sound velocity during reconstruction of the individual $2-\mathrm{D}$ projection images might reduce this artifact. Due to long measurement times, the water temperature increased during data acquisition. Since the speed of sound depends on the water temperature, it is necessary to adjust the speed of sound for the reconstruction of each 2-D projection image.

\subsection{Three-Dimensional Imaging of an Ant}

For demonstrating the ability of imaging small objects and to show the spatial resolution of the integrating fiber-based line detectors, we chose a dead ant for tomography. Due to the ant's dark color, the excitation laser at a wavelength $532 \mathrm{~nm}$ is strongly absorbed. The body of the ant has a length of approximately $6 \mathrm{~mm}$ and varying diameter between $2 \mathrm{~mm}$ (metasoma) down to less than $1 \mathrm{~mm}$ (between caput and mesosoma). The length of the trotters varies between $3 \mathrm{~mm}$ and $5 \mathrm{~mm}$, with diameters of approximately $200 \mu \mathrm{m}$ to $350 \mu \mathrm{m}$. The smaller feelers have a length of $4 \mathrm{~mm}$ and diameters between $160 \mu \mathrm{m}$ and $200 \mu \mathrm{m}$. For clarification, a photograph of the ant next to a ruler can be seen in Fig. 7(a), and a scheme of the anatomy of an ant is depicted in Fig. 7(b). 

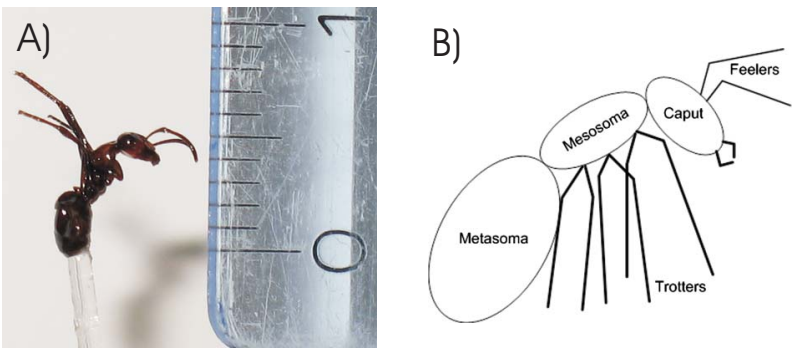

Fig. 7 On the left, a photograph of the ant can be seen. For clarification of proportions, the ant was photographed next to a ruler. The ant is mounted on a nylon rod and fixed by spraying a liquid band-aid (spray plaster) onto the surface. On the right, a schematic of the anatomy of an ant is depicted. The essential parts of the body are labeled.

The ant was mounted on a nylon rod, because nylon does not generate photoacoustic signals at the excitation wavelength. To avoid movement of the ant during imaging (e.g., bending of feelers or trotters), we covered it with five layers of water-resistant liquid band-aid (spray plaster). The trotters unfortunately were glued together, as can be seen in the photograph of the sample (see Fig. 7). Subsequently, the object was immersed in a water-filled tank without any scattering medium.

Excitation parameters (wavelength, pulse duration, repetition rate, laser beam diameter, and energy density) were the same as used for imaging the bristle knot, as described earlier (see Sec. 4.1).

Since we estimated an achievable resolution of $110 \mu \mathrm{m}$ using 262 detector positions, to save time, we decided to use only 200 detector positions for imaging the ant. This amount of detector positions on a circle with a radius of $10 \mathrm{~mm}$ results in an estimated resolution of approximately $140 \mu \mathrm{m}$, which is adequate for the ant with structures of $160 \mu \mathrm{m}$ and more. For the same reasons as mentioned for the bristle knot, only 25 projections with an angle increment of $7.2 \mathrm{deg}$ were acquired to calculate the initial energy distribution. A timereversal reconstruction algorithm (see Sec. 3) was used to derive the projection images at each angle.

In Fig. 8, a projection image of the ant taken at $0 \mathrm{deg}$ can be seen. For reconstruction, we used original data without any signal processing — no filtering or smoothing was applied. The bright spot in the center and the somewhat lower contrast at the regions away from the center attract attention. The photoacoustic signal is proportional to the local energy density times the Grüneisen coefficient. ${ }^{7}$ Thus, absorption should be approximately the same at all parts of the ant. But due to the Gaussian intensity profile of the excitation laser, regions away from the center create weaker signals. All the light (at the excitation wavelength of $532 \mathrm{~nm}$ ) is absorbed in the outer surface, and no penetration of light into the ant occurs. Thus, only imaging of the outer contour of the ant is possible. To acquire information from the inner structure of the ant, it would be necessary to change the excitation wavelength to the near infrared. ${ }^{7}$ Nevertheless, one is able to distinguish between the head (caput), body (mesosoma and metasoma), trotters, and feelers.

Although the 3-D image is calculated from only 25 projections, the ant is well-resolved, as depicted in Fig. 9. For visu-

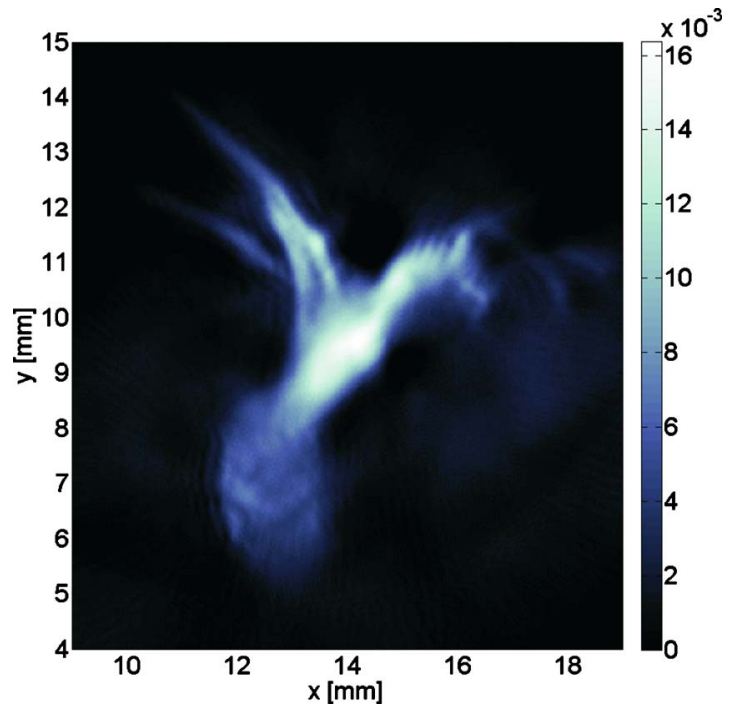

Fig. 8 Two-dimensional projection image of the ant at 0 deg. Photoacoustic signals were acquired at 200 detector positions arranged in a circle around the ant, as depicted in Fig. 5.

alization purposes, only half of the ant is displayed. Because of the shallow penetration depth of light at the excitation wavelength, only the surface of the ant can be reconstructed. Stripe artifacts in the reconstructed volume, as shown in Fig. 10 , arise from the low number of projections used. Figure 10 shows a transverse slice of the ant at a height where the caput and the feelers can be recognized. Increasing the amount of $2-\mathrm{D}$ projection images would reduce the stripe artifacts ${ }^{26}$ significantly but would prolong the measurement time.

From the 3-D reconstruction of the ant, one can see that we were able to reconstruct the fine feelers with a diameter vary-

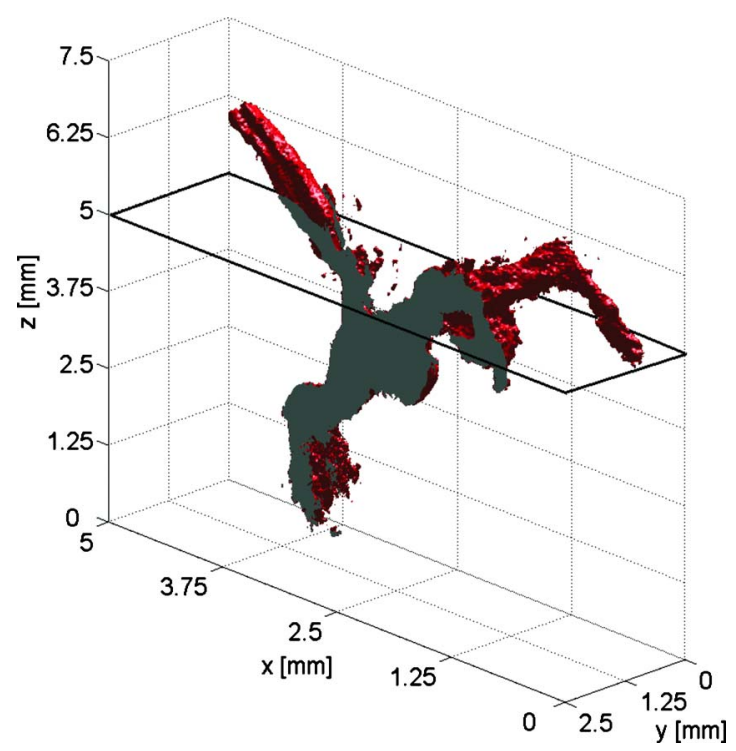

Fig. 9 Three-dimensional reconstruction of the ant from 25 projections, each consisting of 200 detector positions. The volume is sliced in approximately the middle of the ant. The head (caput) with one feeler, the body (mesosoma) with two superposed trotters and the metasoma can be seen clearly. The black slice indicates the height at which the transverse transection in Fig. 10 is taken. 


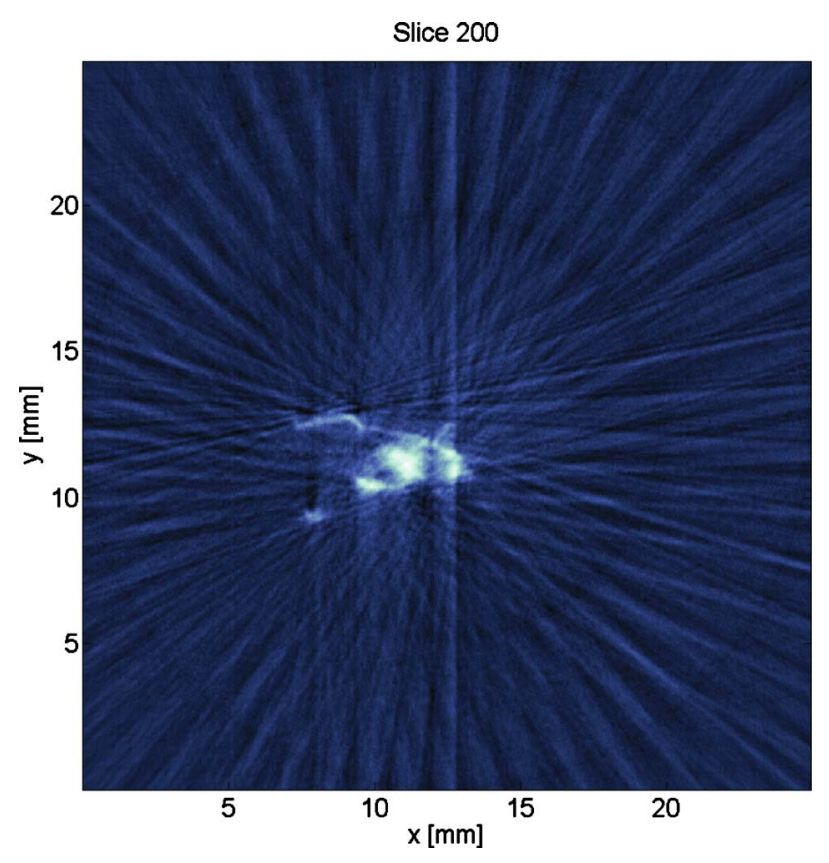

Fig. 10 Due to fewer projection images stripe artifacts occur in the reconstructed volume, as can be seen in the transverse section of the ant. Increasing the amount of projection images would reduce stripe artifacts and increase the spatial resolution. This transverse section was taken at a height as indicated in Fig. 9.

ing between 160 and $200 \mu \mathrm{m}$ and trotters with a diameter varying between 200 and $350 \mu \mathrm{m}$ quite well. Compared to other detection approaches used for photoacoustic imaging, this is a good value. Due to the nonuniform illumination, the lower part of the ant (metasoma) is not well reconstructed. This can be improved by using a beam homogenizer or a scattering medium to embed the object. Preliminary measurements of simple objects cast in polyvinyl alcohol (PVA) showed promising results. ${ }^{20}$ Because of problems casting the ant into PVA (air bubbles adhering between the body and the trotters), we carried out the first measurements using the bare ant without the advantage of a scattering medium.

\section{Conclusion and Outlook}

We presented first 3-D photoacoustic images measured with fiber-based line detectors. By using this type of detector, we were able to measure not only simple objects like a bristle knot but also more complex objects such as an ant. The resolution was high enough to reconstruct fine structures of the ant like the feelers (varying between 160 and $200 \mu \mathrm{m}$ ) or the trotters (varying between 200 and $350 \mu \mathrm{m}$ ). Thus, we could show the capability of this novel type of detectors for photoacoustic imaging in biology and medicine. These first threedimensional measurements showed the potential for future developments. For example, the illumination can be improved by using beam homogenizers. A flat beam instead of a Gaussian beam profile should lead to better image quality. Also the use of scattering media such as polyvinyl alcohol or Intralipid for embedding the sample should homogenize the illumination and therefore lead to better resolved structures. By combining the advantages of a GOF Fabry-Pérot detector (small core diameter, increased sensitivity because of the Bragg gratings) and a POF Mach-Zehnder interferometer (better impedance matching, lower Young's modulus), one could increase the sensitivity considerably. One goal is to realize fiber Bragg gratings in a perfluorinated polymer fiber with a small core diameter. This would increase the sensitivity while keeping the resolution high.

Imaging of other biological samples will be a subsequent step for fiber-based line detectors for photoacoustic imaging. For that purpose, it will be necessary to accelerate the measurement time. This can be done either by using more sensitive detectors, which would reduce the need for averaging, or by using multiple detectors. It also turned out that not as many detector positions as used in the experiments are necessary. Instead of the large amount of detector positions, more projections should be measured to reduce stripe artifacts.

\section{Acknowledgments}

This work has been supported by the Austrian Science Fund (FWF), Project Nos. S10502-N20, S10503-N20, and L418N20; by the European Regional Development Fund (EFRE) in the framework of the EU-program Regio 13; and by the federal state of Upper Austria.

\section{References}

1. L. V. Wang, "Prospects of photoacoustic tomography," Med. Phys. 35, 5758-5767 (2008)

2. S. Manohar, S. E. G. Vaartjes, J. M. van Hespen, J. Klaase, F. van den Engh, W. Steenbergen, and T. G. van Leeuwen, "Initial results of in vivo non-invasive cancer imaging in the human breast using nearinfrared photoacoustics," Opt. Express 15, 12277-12285 (2007).

3. A. G. Bell, "On the production and reproduction of sound by light: the photophone," Am. J. Sci. 20, 305-324 (1880).

4. M. Haltmeier, O. Scherzer, P. Burgholzer, and G. Paltauf, "Thermoacoustic computed tomography with large planar receivers," Inverse Probl. 20, 1663-1673 (2004).

5. P. Burgholzer, C. Hofer, G. Paltauf, M. Haltmeier, and O. Scherzer, "Thermoacoustic tomography with integrating area and line detectors," IEEE Trans. Ultrason. Ferroelectr. Freq. Control 52, 1577$1583(2005)$

6. G. Paltauf, R. Nuster, M. Haltmeier, and P. Burgholzer, "Photoacoustic tomography using a Mach-Zehnder interferometer as acoustic line detector," Appl. Opt. 46, 3352-3358 (2006).

7. M. Xu and L. Wang, "Photoacoustic imaging in biomedicine," Rev. Sci. Instrum. 77, 041101 (2006).

8. P. Burgholzer, G. J. Matt, M. Haltmeier, and G. Paltauf, "Exact and approximative imaging methods for photoacoustic tomography using an arbitrary detection surface," Phys. Rev. E 75, 046706 (2007).

9. H. Grün, G. Paltauf, M. Haltmeier, and P. Burgholzer, "Photoacoustic tomography using a fiber based Fabry-Perot interferometer as an integrating line detector and image reconstruction by model-based time reversal method," in European Conferences on Biomedical Optics: Novel Optical Instrumentation for Biomedical Applications III, edited by C. D. Depeursinge, Proc. SPIE 6631, 663107 (2007).

10. M. Fink, "Time reversal of ultrasonic fields-Part I: Basic principles," IEEE Trans. Ultrason. Ferroelectr. Freq. Control 39, 555566 (1992).

11. M. Holotta, R. Esterhammer, P. Torbica, J. Völkl, C. Kremser, W. Jaschke, H. Grossauer, M. Haltmeier, O. Scherzer, R. Nuster, et al. "Photoacoustic tomography of small animals and organs," Proc. VIPImage - 2nd Eccomas Thematic Conf. on Computational Vision and Processing, Porto, Portugal, Oct. 14-16, 2009, pp. 25-29, CRC Press/Balkema, Leiden, The Netherlands (2009).

12. E. Z. Zhang, J. G. Laufer, R. B. Pedley, and P. C. Beard, "In vivo high-resolution 3D photoacoustic imaging of superficial vascular anatomy," Phys. Med. Biol. 54, 1035-1046 (2009).

13. T. Berer, H. Grün, C. Hofer, and P. Burgholzer, "Photoacoustic microscopy with large integrating optical annular detectors," in European Conferences on Biomedical Optics: Novel Optical Instrumenta- 
tion for Biomedical Applications IV, edited by C. D. Depeursinge, Proc. SPIE 7371, 73710X (2009).

14. G. Zangerl, O. Scherzer, and M. Haltmeier, "Circular integrating detectors in photo- and thermoacoustic tomography," Inverse Probl. Sci. Eng. 17, 133-142 (2009).

15. P. Burgholzer, C. Hofer, G. Paltauf, M. Haltmeier, and O. Scherzer, "Thermoacoustic tomography using integrating detectors," in European Conferences on Biomedical Optics: Novel Optical Instrumentation for Biomedical Applications II, edited by C. D. Depeursinge, Proc. SPIE 5864, 586403 (2005).

16. R. Nuster, S. Gratt, K. Passler, H. Grün, T. Berer, P. Burgholzer, and G. Paltauf, "Comparison of optical and piezoelectric integration line detectors," in Biomedical Optics: Photons Plus Ultrasound: Imaging and Sensing 2009, edited by A. A. Oraevsky and L. H. Wang, Proc. SPIE 7177, 71770T (2009).

17. G. Paltauf, R. Nuster, and P. Burgholzer, "Characterization of integrating ultrasound detectors for photoacoustic tomography," J. Appl. Phys. 105, 102026 (2009).

18. G. Paltauf, P. Burgholzer, M. Haltmeier, and O. Scherzer, "Thermoacoustic tomography using optical line detection," in European Conferences on Biomedical Optics: Novel Optical Instrumentation for Biomedical Applications II, edited by C. D. Depeursinge, Proc. SPIE 5864, 586402 (2005)

19. H. Grün, T. Berer, A. Hochreiner, R. Nuster, G. Paltauf, and P. Burgholzer, "Photoacoustic imaging with integrating line detectors," in Medical Imaging: Ultrasonic Imaging and Signal Processing, edited by S. McAleavey and J. D'hooge, Proc. SPIE 7265, 72650K (2009).

20. H. Grün, T. Berer, R. Nuster, G. Paltauf, and P. Burgholzer, "Fiberbased detectors for photoacoustic imaging," in European Conferences on Biomedical Optics: Novel Optical Instrumentation for Biomedical Applications IV, edited by C. D. Depeursinge, Proc. SPIE 7371, 73710T (2009).

21. R. Nuster, M. Holotta, H. Grossauer, P. Burgholzer, and G. Paltauf, "Photoacoustic micro-tomography using interferometric detection," J. Biomed. Opt. 15, 021307 (2010).

22. G. Paltauf, R. Nuster, K. Passler, M. Haltmeier, and P. Burgholzer, "Optimizing image resolution in three-dimensional photoacoustic tomography with line detectors," in Biomedical Optics: Photons Plus Ultrasound: Imaging and Sensing 2008, edited by A. A. Oraevsky and L. H. Wang, Proc. SPIE 6856, 685621 (2008).

23. S. Kiesel, K. Peters, T. Hassan, and M. Kowalsky, "Behavior of intrinsic polymer optical fibre sensor for large-strain," Meas. Sci. Technol. 18, 3144-3154 (2007).

24. M. Xu and L. Wang, "Universal back-projection algorithm for photoacoustic computed tomography," Phys. Rev. E 71, 016706 (2005).

25. K. P. Köstli, M. Frenz, H. Bebie, and H. P. Weber, "Temporal backward projection of optoacoustic pressure transients using Fourier transform methods," Phys. Med. Biol. 46, 1863-1872 (2001).

26. A. C. Kak and M. Slaney, "Principles of computerized tomographic imaging," Principles of Computerized Tomographic Imaging, IEEE Press, New York (1988).

27. M. Fink, "Time reversed acoustics," Phys. Today 50, 34-40 (1997).

28. H. Grün, R. Nuster, G. Paltauf, M. Haltmeier, and P. Burgholzer, "Photoacoustic tomography of heterogeneous media using modelbased time reversal method," in Biomedical Optics: Photons Plus Ultrasound: Imaging and Sensing 2008, edited by A. A. Oraevsky and L. H. Wang, Proc. SPIE 6856, 685620 (2008). 